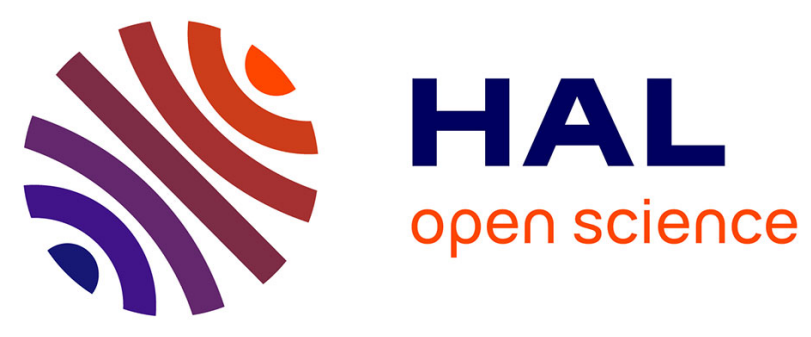

\title{
Adaptive Mode Selection in Cognitive Buffer-Aided Full-Duplex Relay Networks with Imperfect Self-Interference Cancellation for Power and Delay Limited Cases
}

Mostafa Darabi, Nima Namvar, Behrouz Maham, Walid Saad, Merouane

Debbah

\section{To cite this version:}

Mostafa Darabi, Nima Namvar, Behrouz Maham, Walid Saad, Merouane Debbah. Adaptive Mode Selection in Cognitive Buffer-Aided Full-Duplex Relay Networks with Imperfect Self-Interference Cancellation for Power and Delay Limited Cases. 2017 IEEE International Conference on Communications Workshops (ICC Workshops), May 2017, Paris, France. 10.1109/iccw.2017.7962776 . hal-02019172

\section{HAL Id: hal-02019172}

https://hal-centralesupelec.archives-ouvertes.fr/hal-02019172

Submitted on 14 Feb 2019

HAL is a multi-disciplinary open access archive for the deposit and dissemination of scientific research documents, whether they are published or not. The documents may come from teaching and research institutions in France or abroad, or from public or private research centers.
L'archive ouverte pluridisciplinaire HAL, est destinée au dépôt et à la diffusion de documents scientifiques de niveau recherche, publiés ou non, émanant des établissements d'enseignement et de recherche français ou étrangers, des laboratoires publics ou privés. 


\title{
Adaptive Mode Selection in Cognitive Buffer-Aided Full-Duplex Relay Networks with Imperfect Self-Interference Cancellation for Power and Delay Limited Cases
}

\author{
Mostafa Darabi ${ }^{\dagger}$, Nima Namvar ${ }^{\ddagger}$, Behrouz Maham ${ }^{\sharp}$, Walid Saad ${ }^{\S}$, and Merouane Debbah ${ }^{\natural b}$ \\ ${ }^{\dagger}$ School of ECE, College of Engineering, University of Tehran, Iran \\ ${ }^{\ddagger}$ Department of Electrical and Computer Engineering, North Carolina A\&T State University, USA \\ \#Department of Electrical and Electronic Engineering, School of Engineering, Nazarbayev University, Astana, Kazakhstan \\ ${ }^{\S}$ Wireless@ VT, Bradley Department of Electrical and Computer Engineering, Blacksburg, VA 24060, USA \\ ${ }^{\natural}$ Large Networks and Systems Group (LANEAS), CentraleSuplec, Universit Paris-Saclay, 91192 Gif-sur-Yvette, France \\ ${ }^{b}$ Mathematical and Algorithmic Sciences Lab, Huawei France R\&D, Paris, France \\ emails: mostafa.darabi@ut.ac.ir,nnamvar@aggies.ncat.edu,bmaham@ut.ac.ir, walids@vt.edu, merouane.debbah@supelec.fr
}

\begin{abstract}
In this paper, a cognitive radio network is considered in which the secondary network ( $\mathrm{SN}$ ) consists of a source, a buffer-aided full-duplex decode-and-forward relay, and a destination, underlaid over a primary network (PN). An imperfect self-interference (SI) cancellation is assumed at the secondary relay $(\mathrm{SR})$, such that the SI power is proportional to the transmit power of the SR. For the SN with limited power expenditure, a novel joint mode selection and power allocation policy is proposed to maximize the secondary throughput under the constraints of secondary power consumption and a limited average induced interference power at the primary destination. For delay sensitive SN applications, a statistical delay constraint is imposed in which the queue length at the SR can only exceed a specified threshold with a limited probability. In the two proposed policies, the SN decides optimally when to operate in half duplex mode and/or in full duplex mode, and be silent. To avoid data loss in the SN, buffer is used at the SR for data storage. Simulation results show that, for a given interference threshold and statistical delay constraint, the proposed policy outperforms the non-buffer full-duplex, buffer-aided half-duplex, and non-buffer half-duplex policies in terms of the average secondary throughput, the average secondary delay, and the secondary power expenditure.
\end{abstract}

Index Terms-Full duplex relaying, buffer-aided relaying, cognitive radio networks, power allocation, statistical delay constraint.

\section{INTRODUCTION}

By enabling simultaneous transmission and reception over the same frequency, full duplex (FD) communications promises to yield significant performance gains over classical half-duplex (HD) wireless networks [1]. Recently, practical implementation of FD communication has sparked an enormous interest in FD communication both in research and industry [2]. However, the benefits of FD communication come at the price of several technical challenges. Indeed, the performance of an FD node is typically impaired by selfinterference (SI), which occurs due to the energy leakage from the transmitter to the receiver of an FD node. Therefore, FD relaying with imperfect SI has attracted research attention recently. In FD relaying with the imperfect SI cancellation, the non-zero residual SI is proportional to the transmit power of the relay [3].

Buffer-aided relaying was first proposed in the HD relay networks in order to relax the predefined transmission-reception schedule of the conventional HD relaying [4], [5]. More specifically, in buffer-aided relaying, in each time slot, the mode of the HD relay is adaptively selected to be either transmission or reception based on the involved channel qualities. In [6], it is shown that buffer-aided relaying can be generalized to the FD relay networks in order to improve the performance. In particular, in contrast to the conventional FD relaying with the simultaneous transmission and reception at the relay, in buffer-aided FD relaying, the FD relay should adaptively choose whether to transmit, receive, or simultaneously transmit and receive data based on the qualities of the involved links [6]. In [7]-[9], buffer-aided relaying policies were proposed for improving the performance of the primary network (PN) and the secondary network $(\mathrm{SN})$ in cognitive radio networks (CRN). In [7] and [8], a heuristic buffer-aided relay selection policy is introduced for the SN with multiple HD relays, where in each time slot, the best relay is selected to transmit data to the secondary destination (SD) under the condition of limited instantaneous interference at the primary destination (PD). In [9], a buffer-aided link selection policy was introduced for the CRN with the HD secondary relay (SR) to maximize the secondary throughput.

Despite the performance gains achieved in the buffer-aided relay networks, one challenge in such networks is the introduced delay that can impair the quality of service (QoS) for the delay intolerant applications. The proposed protocols in [7][9], improve the secondary throughput with the expense of an unbounded delay in the HD SN. Due to the deep fades of a wireless channel, imposing deterministic delay bound usually results in high power consumption. On the other hand, a small probability of delay bound infringement can be withstood by the most delay sensitive applications such as multimedia streaming, and video telephony. In [10], a statistical delay constraint is considered in which a certain bound of the queue length at the relay is allowed to be violated within a maximum acceptable probability. Similarly, the statistical delay constraint can be applied for the delay sensitive CRNs. In our proposed policy, in order to guarantee the secondary delay QoS, we impose the statistical delay constraint in the SN.

The main contributions of this paper are two novel mode 
selection protocols for buffer-aided FD CRN that allow the maximization of the secondary throughput under constraint on the average interference (A-INT) power at the PD for both power and delay limited cases. In contrast to the proposed policies in [7]-[9], in our approach, the SR is an FD node with imperfect SI cancellation in which the SI power is proportional to the transmitted power at the SR. For a power limited SN, a joint mode selection and power allocation scheme is proposed to maximize the secondary throughput under the constraints of secondary power expenditure and a limited induced secondary interference at the PN. Furthermore, for delay sensitive SN applications, a statistical delay constraint is imposed in which the length of queue at the SR can be over a fixed threshold within a limited probability. In particular, in the two proposed protocols, the SN can optimally decide when to operate in HD mode and/or in FD mode, and be silent. Simulation results show that our proposed adaptive mode selection policies yield considerable secondary throughput gains, low secondary power expenditure, and low secondary average delay compared to the buffer-aided HD in [9], non-buffer FD, and non-buffer HD policies in the SN.

\section{SySTEM MODEL}

Consider a cooperative CRN, shown in Fig. 1, in which the PN consists of a primary source (PS) and a PD, and the SN includes a SD that can receive data of a secondary source (SS) only via an FD SR. Time is divided into equal length time slots. All wireless channels exhibit an additive white Gaussian noise (AWGN) with zero mean and unit variance and are impaired by flat block fading. At time slot $i, h_{s r}(i)$, $h_{r d}(i), g_{p 1}(i)$, and $g_{p 2}(i)$ denote the channel coefficients between the SS and the SR, between the SR and the SD, between the PS and the SR, and between the PS and the SD, respectively. The SI channel at the SR has a channel gain of $g_{r r}(i)$, and the channel coefficients between the SS and the $\mathrm{PD}$, between the SR and the PD, and between the PS and PD are given by $g_{s 1}(i), g_{s 2}(i)$, and $h_{p}(i)$, respectively. The channel coefficients are considered to be stationary and ergodic stochastic processes. In our model, the primary transmit power is assumed to be fixed and is denoted $P_{p}$. The transmit powers of the SS and the SR during slot $i$ are denoted by $P_{s}(i)$ and $P_{r}(i)$, respectively. $\bar{P}_{s}$ and $\bar{P}_{r}$ denote the average transmit power of the SS and the SR, respectively. $\bar{P}_{i n t}$ is the A-INT power induced at the PD.

To prevent data loss at the SN, the SR has equipped with a buffer. $Q(i)$ captures the amount of normalized information in bits/symbol saved in the buffer of the SR at the end of time slot $i$. At the SR, the received data is decoded, stored in the buffer, and finally retransmitted to the SD. In our proposed protocols, the primary interference experienced at the SR and $\mathrm{SD}$ is treated as noise. Let $C_{s r}^{h}(i)$ and $C_{r d}^{h}(i)$ be, respectively, the capacity of the SS-SR and SR-SD links in the HD mode. In case of available channel state information (CSI) of the interference channels, $C_{s r}^{h}(i)$ and $C_{r d}^{h}(i)$ can be written as

$$
C_{s r}^{h}(i)=\log _{2}\left(1+\frac{P_{s}(i)\left|h_{s r}(i)\right|^{2}}{1+P_{p}\left|g_{p 1}(i)\right|^{2}}\right),
$$

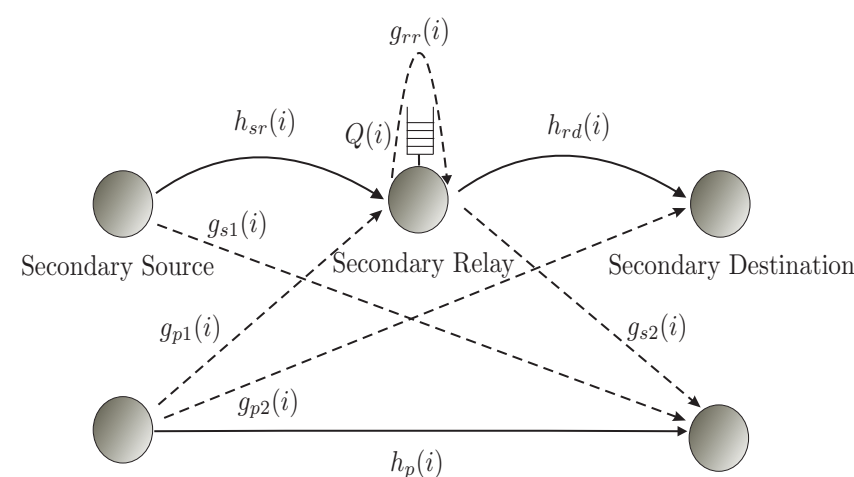

Primary Source

Signal

Primary Destination

Fig. 1. The considered buffer-aided FD CRN.

$$
C_{r d}^{h}(i)=\log _{2}\left(1+\frac{P_{r}(i)\left|h_{r d}(i)\right|^{2}}{1+P_{p}\left|g_{p 2}(i)\right|^{2}}\right) .
$$

In the FD mode, the SI power at the SR is proportional to the transmit power at the SR, which yields the following SS-SR link capacity:

$$
C_{s r}^{f}(i)=\log _{2}\left(1+\frac{P_{s}(i)\left|h_{s r}(i)\right|^{2}}{1+P_{r}(i)\left|g_{r r}(i)\right|^{2}+P_{p}\left|g_{p 1}(i)\right|^{2}}\right) .
$$

Further, the capacity of the SR-SD link in the FD mode is similar to the HD mode, i.e., $C_{r d}^{f}(i)=C_{r d}^{h}(i)=C_{r d}(i)$.

In our proposed protocol, during each time slot, the mode of operation of the $\mathrm{SN}$ is selected as either silent, $\mathrm{HD}$, and/or FD such that the secondary throughput is maximized under the constraint of a limited interference induced to the PD. For the power limited and delay intolerant SN, a limited average secondary power consumption and a low statistical delay constraints are also imposed, respectively. The binary variables $q_{1}(i), q_{2}(i), q_{3}(i) \in\{0,1\}$ are used for SN mode selection. Here, if $q_{1}(i)=1$, the SN operates in HD mode and the SSSR link is selected for data transmission at slot $i$ allowing the SS to transmit with maximum rate $R_{s r}(i)=C_{s r}^{h}(i)$. At the SR, data is decoded and stored in the buffer, and thus, at the end of $i$-th time slot, the buffer at the SR stores $Q(i)=Q(i-1)+R_{s r}(i)$ bits/symbol of information. In contrast, if we have $q_{2}(i)=1$, then the SN operates in HD mode and data is transmitted via the SR-SD link with the data rate of $R_{r d}(i)=\min \left\{Q(i-1), C_{r d}(i)\right\}$. Here, the amount of information stored in the buffer decreases to $Q(i)=Q(i-1)-R_{r d}(i)$ bits/symbol. Similarly, if $q_{3}(i)=1$, the SN operates in FD mode and both of the SS and the SR transmit data with the rate of $R_{s r}(i)=C_{s r}^{f}(i)$ and $R_{r d}(i)=\min \left\{Q(i-1), C_{r d}(i)\right\}$, respectively, and thus, at the end of $i$-th time slot, the buffer at the SR stores $Q(i)=Q(i-1)+R_{s r}(i)-R_{r d}(i)$ bits/symbol of information.

\section{Proposed Buffer-Aided Mode Selection PROTOCOLS}

Here, after investigating the secondary achievable average rates, the average power of interference at the PD, and the secondary statistical delay constraint, two optimization problems are formulated to maximize the secondary throughput for 
the limited power and delay intolerant cases. Then, a bufferaided joint mode selection and power allocation protocol and a buffer-aided mode selection policy with statistical delay constraint are derived for an $\mathrm{SN}$ with the limited power budget and delay sensitive applications.

\section{A. Achievable Average Rates of the SN}

Under the assumption of an infinite number of slots and a continuous stream of information at the SS, the average transmission rates of the SS-SR link and the SR-SD link, denoted by $\bar{R}_{s r}$ and $\bar{R}_{r d}$, respectively, will be:

$$
\begin{aligned}
& \bar{R}_{s r}=\lim _{N \rightarrow \infty} \frac{1}{N} \sum_{i=1}^{N} q_{1}(i) C_{s r}^{h}(i)+q_{3}(i) C_{s r}^{f}(i) \\
& \bar{R}_{r d}=\lim _{N \rightarrow \infty} \frac{1}{N} \sum_{i=1}^{N}\left(q_{2}(i)+q_{3}(i)\right) \min \left\{C_{r d}(i), Q(i-1)\right\}
\end{aligned}
$$

The buffer of the SR always has enough data to send, and thus, the SR has the following average processing rate

$$
\bar{R}_{r d}^{*}=\lim _{N \rightarrow \infty} \frac{1}{N} \sum_{i=1}^{N}\left(q_{2}(i)+q_{3}(i)\right) C_{r d}(i) .
$$

For an infinite-size buffer, if the average input rate and the average processing rate are equal, i.e., $\bar{R}_{s r}=\bar{R}_{r d}^{*}$, the queue is rate stable, and $\bar{R}_{r d}=\bar{R}_{r d}^{*}$ holds [5].

\section{B. CSI Assumption and Induced Interference Power at the PD}

In our proposed mode selection protocols, it is assumed that the perfect instantaneous CSI of the secondary channels is available. As such, the SR receives pilot signals from the SS and SD and estimates the CSI of the SS-SR and SR-SD links. In this paper, two cases of available instantaneous CSI of the interference links (I-CSI-I) and available statistical CSI of the interference links (S-CSI-I) are considered. For the A-INT power at the $\mathrm{PD}$, we have

$$
\bar{P}_{i n t}=\left\{\begin{array}{cc}
\frac{1}{N} \sum_{i=1}^{N}\left(q_{1}(i) P_{s}(i)\left|g_{s 1}(i)\right|^{2}+q_{2}(i) P_{r}(i)\left|g_{s 2}(i)\right|^{2}\right. \\
\left.\quad+q_{3}(i)\left(P_{s}(i)\left|g_{s 1}(i)\right|^{2}+P_{r}(i)\left|g_{s 2}(i)\right|^{2}\right)\right), & \text { I-CSI-I, } \\
\frac{\Psi_{1}}{N} \sum_{i=1}^{N} P_{s}(i)\left(q_{1}(i)+q_{3}(i)\right) & \\
+\frac{\Psi_{2}}{N} \sum_{i=1}^{N} P_{r}(i)\left(q_{2}(i)+q_{3}(i)\right), & \text { S-CSI-I, }
\end{array}\right.
$$

in which $\Psi_{1}=\mathbb{E}\left\{\left|g_{s 1}(i)\right|^{2}\right\}, \Psi_{2}=\mathbb{E}\left\{\left|g_{s 2}(i)\right|^{2} \mid\right\}$, and $\mathbb{E}\{\cdot\}$ is the expectation.

\section{Statistical Delay Constraint at the SN}

Given a stable queue at the SR, a steady-state queue length distribution can be considered for the SR. Let $Q$ be the steady state queue length distribution of the SR. The statistical delay constraint in the SN can be defined as the maximum allowable queue length outage probability [10]:

$$
\operatorname{Pr}\left\{Q>Q^{\max }\right\} \leq \xi
$$

where $0<Q^{\max }$ and $0<\xi \leq 1$ hold. Assume that the queue at the SR satisfies the Gartner-Ellis limit, i.e., the following logarithmic moment generating functions (LMGF) for the arrival and service processes of the $\mathrm{SR}, \Upsilon_{R_{s r}(i)}(\theta)$ and $\Upsilon_{R_{r d}(i)}(\theta)$, exist for all $\theta>0$.

$$
\Upsilon_{R_{s r}(i)}(\theta)=\log \mathbb{E}\left[e^{\theta R_{s r}(i)}\right], \quad \Upsilon_{R_{r d}(i)}(\theta)=\log \mathbb{E}\left[e^{\theta R_{r d}(i)}\right] .
$$

If for a unique achievable delay exponent $\theta>0$, $\Upsilon_{R_{s r}(i)}(\theta)+\Upsilon_{R_{r d}(i)}(-\theta)=0$ holds, for sufficiently large $x$, the steady state queue length outage probability at the SR can be written as [11]

$$
\operatorname{Pr}\{Q>x\}=e^{-\theta x} \text {. }
$$

For satisfy the statistical delay constraint in (8) and achieve the maximum secondary throughput, we must have:

$$
\theta=\frac{-1}{Q^{\max }}\left(1+\mathcal{W}_{-1}\left(\frac{-\xi}{e}\right)\right)
$$

with $\mathcal{W}_{-1}($.$) being the real branch of Lambert \mathrm{W}$ function. Therefore, $\Upsilon_{R_{s r}(i)}(\theta)=-\Upsilon_{R_{r d}(i)}(-\theta)$ can be used as the statistical delay constraint of the SN instead of (8). By some mathematical manipulations, $\Upsilon_{R_{s r}(i)}(\theta)=-\Upsilon_{R_{r d}(i)}(-\theta)$ results in $\mathbb{E}\left\{e^{\theta R_{s r}(i)}\right\}=\frac{1}{\mathbb{E}\left\{e^{-\theta R_{r d}(i)}\right\}}$.

\section{Buffer-Aided Joint Mode Selection and Power Allocation Protocol}

In a power limited $\mathrm{SN}$, the secondary throughput can be maximized by solving the following optimization problem.

$$
\begin{aligned}
\max _{\boldsymbol{q}(i), P_{s}(i), P_{r}(i) \forall i} & \bar{R}_{r d}^{*} \\
\text { subject to C1: } & \bar{P}_{\text {int }} \leq I_{t h r} \\
\mathrm{C} 2: & \bar{R}_{s r}=\bar{R}_{r d}^{*} \\
\mathrm{C} 3: & P_{s}(i), P_{r}(i) \geq 0 \\
\mathrm{C} 4: & \bar{P}_{s}+\bar{P}_{r} \leq P_{b} \\
\text { C5: } & \boldsymbol{q}(i) \in \mathcal{Q}, \forall i,
\end{aligned}
$$

in which $\boldsymbol{q}(i)=\left[q_{1}(i), q_{2}(i), q_{3}(i)\right]$. The induced A-INT power at the PD is limited by constraint $\mathrm{C} 1$ to be lower than an interference threshold $I_{t h r}$. Rate stability of the buffer at the SR is ensured by constraint C2. Constraint C3 ensures that the instantaneous transmit powers at the $\mathrm{SN}$ are positive and constraint $\mathrm{C} 4$ limits the average transmit powers of the SS and the SR to be below a power budget limit, $P_{b}$. In addition, the set of $\mathcal{Q}$ can be written as $\mathcal{Q}=\left\{\left[q_{1}(i), q_{2}(i), q_{(} 3\right)(i)\right] \mid q_{1}(i), q_{2}(i), q_{3}(i) \in\{0,1\} \wedge$ $\left.q_{1}(i)+q_{2}(i)+q_{3}(i) \leq 1\right\}$.

Theorem 1. In a power limited $\mathrm{SN}$ and for both the I-CSI-I and S-CSI-I assumptions, the secondary throughput is maximized by the following optimal sequences of $q_{1}(i)$, $q_{2}(i)$, and $q_{3}(i)$ :

$\boldsymbol{q}(i)= \begin{cases}{[0,0,0],} & \text { if } \Gamma_{1}(i), \Gamma_{2}(i), \Gamma_{3}(i)<0, \\ {[1,0,0],} & \text { if } \Gamma_{1}(i) \geq \Gamma_{2}(i) \geq 0 \vee \Gamma_{1}(i)>0>\Gamma_{2}(i) \\ & \wedge \Gamma_{1}(i) \geq \Gamma_{3}(i) \geq 0 \vee \Gamma_{1}(i)>0>\Gamma_{3}(i), \\ {[0,1,0],} & \text { if } \Gamma_{2}(i)>\Gamma_{1}(i)>0 \vee \Gamma_{2}(i)>0>\Gamma_{1}(i) \\ & \wedge \Gamma_{2}(i) \geq \Gamma_{3}(i) \geq 0 \vee \Gamma_{2}(i)>0>\Gamma_{3}(i), \\ {[0,0,1],} & \text { if } \Gamma_{3}(i)>\Gamma_{1}(i)>0 \vee \Gamma_{3}(i)>0>\Gamma_{1}(i) \\ & \wedge \Gamma_{3}(i) \geq \Gamma_{2}(i) \geq 0 \vee \Gamma_{3}(i)>0>\Gamma_{2}(i),\end{cases}$ 
in which $\Gamma_{1}(i), \Gamma_{2}(i)$, and $\Gamma_{3}(i)$ are as follows:

$$
\begin{gathered}
\Gamma_{1}(i) \triangleq\left\{\begin{array}{l}
\lambda C_{s r}^{h}(i)-\mu P_{s}^{h}(i)\left|g_{s 1}(i)\right|^{2}-\gamma P_{s}^{h}(i), \text { I-CSI-I, } \\
\hat{\lambda} C_{s r}^{h}(i)-\hat{\mu} P_{s}^{h}(i) \Psi_{1}-\hat{\gamma} P_{s}^{h}(i), \quad \text { S-CSI-I, }
\end{array},\right. \\
\Gamma_{2}(i) \triangleq \begin{cases}(1-\lambda) C_{r d}(i)-\mu P_{r}^{h}(i)\left|g_{s 2}(i)\right|^{2}-\gamma P_{r}^{h}(i), \text { I-CSI-I } \\
(1-\hat{\lambda}) C_{r d}(i)-\hat{\mu} P_{r}^{h}(i) \Psi_{2}-\hat{\gamma} P_{r}^{h}(i), \quad \text { S-CSI-I, }\end{cases} \\
\Gamma_{3}(i) \triangleq\left\{\begin{array}{l}
(1-\lambda) C_{r d}(i)-\gamma\left(P_{s}^{f}(i)+P_{r}^{f}(i)\right)+\lambda C_{s r}^{f}(i) \\
-\mu\left(P_{s}^{f}(i)\left|g_{s 1}(i)\right|^{2}+P_{r}^{f}(i)\left|g_{s 2}(i)\right|^{2}\right), \text { I-CSI-I, } \\
(1-\hat{\lambda}) C_{r d}(i)-\hat{\gamma}\left(P_{s}^{f}(i)+P_{r}^{f}(i)\right)+\hat{\lambda} C_{s r}^{f}(i) \\
-\hat{\mu}\left(P_{s}^{f}(i) \Psi_{1}+P_{r}^{f}(i) \Psi_{2}\right),
\end{array}\right.
\end{gathered}
$$

where $P_{s}^{h}(i)$, and $P_{s}^{f}(i)$ are the transmit power of the SS in HD and FD modes, respectively, and $P_{r}^{h}(i)$, and $P_{r}^{f}(i)$ denote the transmit power of the SR in HD and FD modes, respectively. For the transmit powers in the HD mode we have

$$
\begin{aligned}
& P_{s}^{h}(i)=\left\{\begin{array}{l}
{\left[\frac{\lambda}{\ln 2\left(\mu\left|g_{s 1}(i)\right|^{2}+\gamma\right)}-\frac{1+P_{p}\left|g_{p 1}(i)\right|^{2}}{\left|h_{s r}(i)\right|^{2}}\right]^{+}, \text {I-CSI-I, }} \\
{\left[\frac{\lambda}{\ln 2\left(\mu \Psi_{1}+\gamma\right)}-\frac{1+P_{p} \mathbb{E}\left\{\left|g_{p 1}(i)\right|^{2}\right\}}{\left|h_{s r}(i)\right|^{2}}\right]^{+}, \text {S-CSI-I },}
\end{array}\right. \\
& P_{r}^{h}(i)=\left\{\begin{array}{l}
{\left[\frac{1-\lambda}{\ln 2\left(\mu\left|g_{s 2}(i)\right|^{2}+\gamma\right)}-\frac{1+P_{p}\left|g_{p 2}(i)\right|^{2}}{\left|h_{r d}(i)\right|^{2}}\right]^{+}, \text {I-CSI-I, }} \\
{\left[\frac{1-\lambda}{\ln 2\left(\mu \Psi_{2}+\gamma\right)}-\frac{1+P_{p} \mathbb{E}\left\{\left|g_{p 2}(i)\right|^{2}\right\}}{\left|h_{r d}(i)\right|^{2}}\right]^{2}, \text { S-CSI-I },}
\end{array}\right.
\end{aligned}
$$

in which $[x]^{+}=\max x, 0$. The transmit power of the SS and the $\mathrm{SR}$ in the FD are

$$
\begin{aligned}
& \int\left[\frac{\lambda\left|h_{s r}(i)\right|^{2}+\left|g_{r r}(i)\right|^{2}}{\ln 2\left(\mu\left|g_{s 1}(i)\right|^{2}+\gamma\right)\left|h_{s r}(i)\right|^{2}}+\right. \\
& P_{s}^{f}(i)=\left\{\begin{array}{c}
\left.-\frac{1+P_{p}\left|g_{p 1}(i)\right|^{2}}{\left|h_{s r}(i)\right|^{2}+\left|g_{r r}(i)\right|^{2}}\right]^{+}, \text {I-CSI-I } \\
{\left[\lambda\left|h_{s r}(i)\right|^{2}+\mathbb{E}\left\{\left|g_{r r}(i)\right|^{2}\right\}\right.}
\end{array}\right. \\
& {\left[\frac{\left.\lambda\left|h_{s r}(i)\right|^{2}+1 \Psi_{1}+\gamma\right)\left|h_{s r}(i)\right|^{2}}{\ln 2\left(\mu \Psi_{1}\right.}+\right.} \\
& \left.-\frac{1+P_{p} \mathbb{E}\left\{\left|g_{p 1}(i)\right|^{2}\right\}}{\left|h_{s r}(i)\right|^{2}+\mathbb{E}\left\{\left|g_{r r}(i)\right|^{2}\right\}}\right]^{+}, \text {S-CSI-I, } \\
& \int\left[\frac{1-\lambda}{\ln 2\left(\mu\left|g_{s 2}(i)\right|^{2}+\gamma+\left|g_{r r}(i)\right|^{2}\right)}+\right. \\
& P_{r}^{f}(i)=\left\{\begin{array}{c}
\left.-\frac{1+P_{p}\left|g_{p 2}(i)\right|^{2}}{\left|h_{r d}(i)\right|^{2}+\left|g_{r r}(i)\right|^{2}}\right]^{+}, \text {I-CSI-I, } \\
{\left[\frac{1-\lambda}{\ln 2\left(\mu \Psi_{2}+\gamma+\mathbb{E}\left\{\left|g_{r r}(i)\right|^{2}\right\}\right)}+\right.} \\
\left.-\frac{1+P_{p} \mathbb{E}\left\{\left|g_{p 2}(i)\right|^{2}\right\}}{\left|h_{r d}(i)\right|^{2}+\mathbb{E}\left\{\left|g_{r r}(i)\right|^{2}\right\}}\right]^{+}, \text {S-CSI-I. }
\end{array}\right.
\end{aligned}
$$

Proof: Please refer to Appendix A.

The variables $(\mu, \lambda, \gamma)$ and $(\hat{\mu}, \hat{\lambda}, \hat{\gamma})$ are the Lagrangian multipliers for the constraints $(\mathrm{C} 1, \mathrm{C} 2, \mathrm{C} 4)$ in (12) with ICSI-I and S-CSI-I knowledge, respectively. These Lagrangian multipliers depend only on the statistics of the channels, and thus, are constant for a given channel realization. Constants $(\mu, \lambda, \gamma)$ and $(\hat{\mu}, \hat{\lambda}, \hat{\gamma})$, can be found via a three-dimensional search such that constraints $(\mathrm{C} 1, \mathrm{C} 2, \mathrm{C} 4)$ in (12) are satisfied for both the I-CSI-I and S-CSI-I knowledge. By exploiting our proposed buffer-aided joint mode selection and power allocation protocol in (13)-(20), in each time slot, the optimal secondary transmission mode with the respective transmit power is determined in order to maximize the secondary throughput.

\section{E. Buffer-Aided Mode Selection with Statistical Delay Con- straint}

In a delay sensitive $\mathrm{SN}$, the following optimization problem maximizes the secondary throughput constrained on a statistical delay.

$$
\begin{array}{ll}
\max _{\boldsymbol{q}(i) \forall i} & \bar{R}_{r d}^{*} \\
\text { subject to C1: } & \bar{P}_{\text {int }} \leq I_{t h r} \\
\mathrm{C} 2: & \bar{R}_{s r}=\bar{R}_{r d}^{*} \\
\mathrm{C} 3: & \mathbb{E}\left\{e^{\theta R_{s r}(i)}\right\}=\frac{1}{\mathbb{E}\left\{e^{-\theta R_{r d}(i)}\right\}} \\
\mathrm{C} 4: & \boldsymbol{q}(i) \in \mathcal{Q}, \forall i,
\end{array}
$$

where condition $\mathrm{C} 3$ with $\theta=\frac{-1}{Q^{\max }}\left(1+\mathcal{W}_{-1}\left(\frac{-\xi}{e}\right)\right)$ corresponds to the statistical delay constraint introduced in (8).

Theorem 2. In a delay intolerant SN and for both the I-CSI-I and S-CSI-I assumptions, the optimal sequences of $q_{1}(i)$, $q_{2}(i)$, and $q_{3}(i)$ for the secondary throughput maximization with the statistical delay constraint are given by (13) in which $\Gamma_{1}(i), \Gamma_{2}(i)$, and $\Gamma_{3}(i)$ are as follows:

$$
\begin{gathered}
\Gamma_{1}(i) \triangleq\left\{\begin{array}{l}
\left.\lambda C_{s r}^{h}(i)-\mu P_{s}^{h}(i)\left|g_{s 1}(i)\right|^{2}-\rho \theta C_{s r}^{h}(i) e^{\theta C_{s r}^{h}(i)}, \text { I-CSI-I, } 22\right) \\
\hat{\lambda} C_{s r}^{h}(i)-\hat{\mu} P_{s}^{h}(i) \Psi_{1}-\hat{\rho} \theta C_{s r}^{h}(i) e^{\theta C_{s r}^{h}(i),} \text { S-CSI-I, }
\end{array}\right. \\
\Gamma_{2}(i) \triangleq\left\{\begin{array}{l}
(1-\lambda) C_{r d}(i)-\mu P_{r}^{h}(i)\left|g_{s 2}(i)\right|^{2}+\rho \theta N^{2} C_{r d}(i), \text { I-CSI-I, } \\
(1-\hat{\lambda}) C_{r d}(i)-\hat{\mu} P_{r}^{h}(i) \Psi_{2}+\hat{\rho} \theta N^{2} C_{r d}(i), \quad \text { S-CSI-I, }
\end{array}\right. \\
\Gamma_{3}(i) \triangleq\left\{\begin{array}{l}
(1-\lambda) C_{r d}(i)-\mu\left(P_{s}^{f}(i)\left|g_{s 1}(i)\right|^{2}+P_{r}^{f}(i)\left|g_{s 2}(i)\right|^{2}\right) \\
+\lambda C_{s r}^{f}(i)-\rho \theta C_{s r}^{f}(i) e^{\theta C_{s r}^{f}(i)}+\rho \theta N^{2} C_{r d}(i), \text { I-CSI-I, } \\
(1-\hat{\lambda}) C_{r d}(i)-\hat{\mu}\left(P_{s}^{f}(i) \Psi_{1}+P_{r}^{f}(i) \Psi_{2}\right), \\
+\hat{\lambda} C_{s r}^{f}(i)-\hat{\rho} \theta C_{s r}^{f}(i) e^{\theta C_{s r}^{f}(i)}+\hat{\rho} \theta N^{2} C_{r d}(i), \text { S-CSI-I, }
\end{array}\right.
\end{gathered}
$$

in which $\rho$ and $\hat{\rho}$ are the Lagrangian multipliers for the constraint C3 in (21) with I-CSI-I and S-CSI-I knowledge, respectively.

Proof: Can be proven in a similar way as Theorem. 1.

\section{Simulation Results And AnAlysis}

For our simulations, a Rayleigh block fading channel is assumed and we set $P_{p}=10 \mathrm{~dB}, P_{b}=5 \mathrm{~dB}, \mathbb{E}\left\{\left|h_{p}(i)\right|^{2}\right\}=$ $0.1, \mathbb{E}\left\{\left|h_{s r}(i)\right|^{2}\right\}=\mathbb{E}\left\{\left|h_{r d}(i)\right|^{2}\right\}=1, \mathbb{E}\left\{\left|g_{p 1}(i)\right|^{2}\right\}=$ $\mathbb{E}\left\{\left|g_{p 2}(i)\right|^{2}\right\}=0.1$, and $\Psi_{1}=\Psi_{2}=0.1$. This implicitly assumes that the $\mathrm{SN}$ has a small size compared to the PN. The average secondary throughput is shown in Fig. 2 in terms of the interference threshold, $I_{t h r}$, for S-CSI-I and I-CSII assumptions. In this figure, the average throughput of the $\mathrm{SN}$ is shown for our first proposed buffer-aided joint mode selection and power allocation protocol, non-buffer FD SN, buffer-aided HD SN in [9], and non-buffer HD SN policies. As Fig. 2 shows, our first proposed protocol outperform other policies in terms of the average secondary throughput. This 


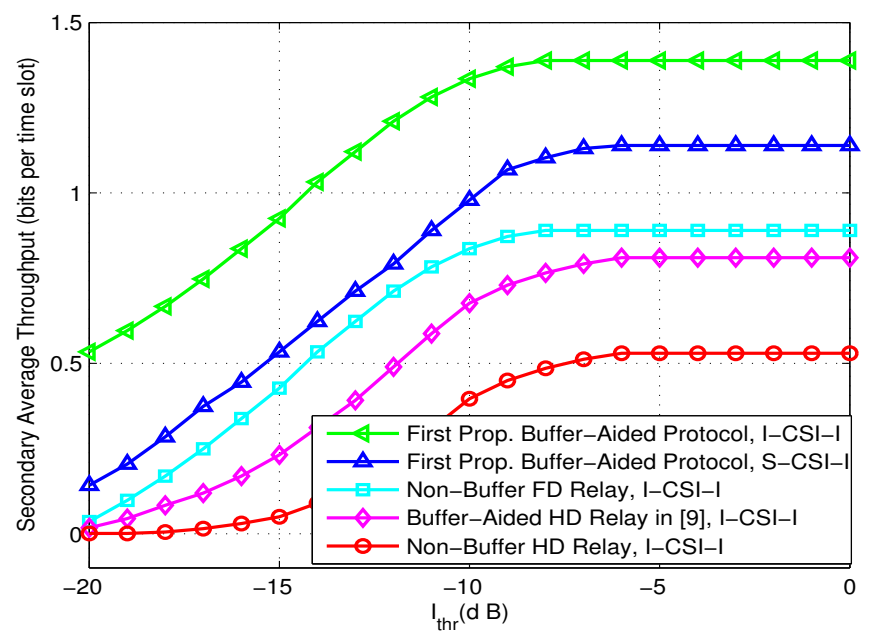

Fig. 2. Average secondary throughput versus $I_{t h r}(\mathrm{~dB})$.

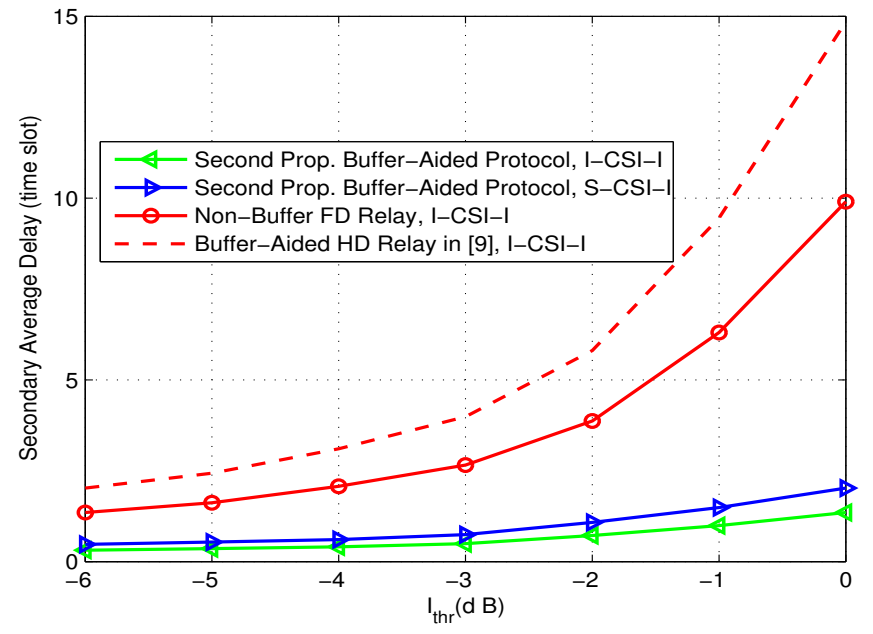

Fig. 3. Average secondary delay versus $I_{t h r}(\mathrm{~dB})$.

improvement reaches up to $55 \%, 75 \%$ and $160 \%$, relative to non-buffer FD SN, buffer-aided HD SN in [9], and non-buffer HD SN policies, respectively.

In Fig. 3, the average delay of the $\mathrm{SN}$ is shown as the interference threshold varies. In Fig. 3, we can see that our second proposed protocol with statistical delay constraint achieves lower average delay compared with non-buffer FD SN, bufferaided HD SN in [9], and non-buffer HD SN policies. Fig. 4 shows the average secondary throughput with respect to the secondary power budget. Fig. 4 also shows that our first proposed protocol has better secondary throughput for the case of a limited power budget at the $\mathrm{SN}$ in comparison with the other policies.

\section{APPENDIX A}

First, consider the case of I-CSI-I. We have an integer program in (12), and hence, to solve it, we relax the binary constraints of $q_{j}(i) \in\{0,1\}, j \in\{1,2,3\}$ into $0 \leq q_{j}(i) \leq 1$. Nevertheless, since the obtained relaxed problem is linear in terms of $q_{j}(i)$, we can find the optimal solution at the boundaries of $0 \leq q_{j}(i) \leq 1$, and thus, by the relaxation we do not lose the optimality. Then, the optimal solution can be found from the Karush-Kuhn-Tucker (KKT) necessary and sufficient conditions for the relaxed linear optimization problem.

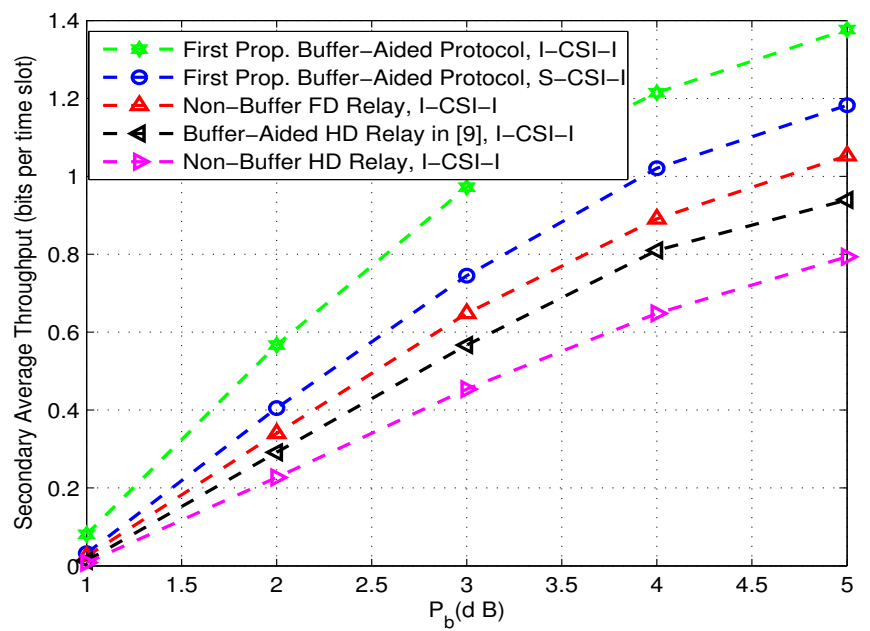

Fig. 4. Average secondary throughput versus $P_{b}(\mathrm{~dB})$.

The relaxed optimization problem has the following Lagrangian function

$$
\begin{aligned}
& \mathcal{L}\left(q_{j}(i), P_{s}(i), P_{r}(i), \mu, \lambda, \nu_{1}, \nu_{2}, \gamma, \alpha_{j}(i), \beta_{j}(i)\right)= \\
& -\bar{R}_{r d}^{*}+\mu\left(\bar{P}_{i n t}-I_{t h r}\right)+\lambda\left(\bar{R}_{r d}^{*}-\bar{R}_{s r}\right)-\sum_{i=1}^{N} \nu_{1}(i) P_{s}(i) \\
& -\sum_{i=1}^{N} \nu_{2}(i) P_{r}(i)+\gamma\left(\bar{P}_{s}+\bar{P}_{r}-P_{b}\right)+\sum_{i=1}^{N} \sum_{j=1}^{3} \alpha_{j}(i)\left(q_{j}(i)-1\right) \\
& -\sum_{i=1}^{N} \sum_{j=1}^{3} \beta_{j}(i) q_{j}(i)+\sum_{i=1}^{N} \varphi(i)\left(q_{1}(i)+q_{2}(i)+q_{3}(i)-1\right),(25)
\end{aligned}
$$

where $\mu, \lambda, \nu_{j}(i), \gamma, \alpha_{j}(i), \beta_{j}(i)$, and $\varphi(i)$ are the Lagrangian multipliers for constraints $\mathrm{C} 1, \mathrm{C} 2, \mathrm{C} 3, \mathrm{C} 4, q_{j}(i) \leq 1$, $q_{j}(i) \geq 0$, and $q_{1}(i)+q_{2}(i)+q_{3}(i) \leq 1$, respectively. Next, we differentiate the Lagrangian function with respect to $q_{1}(i)$, $q_{2}(i)$, and $q_{3}(i)$ and equate it to zero.

$$
\begin{aligned}
& \frac{\partial \mathcal{L}}{\partial q_{1}(i)}=\frac{\mu}{N} P_{s}(i)\left|g_{s 1}(i)\right|^{2}-\frac{\lambda}{N} C_{s r}^{h}(i)+\frac{\gamma}{N} P_{s}(i)+\alpha_{1}(i) \\
& -\beta_{1}(i)+\varphi(i)=0, \\
& \frac{\partial \mathcal{L}}{\partial q_{2}(i)}=\frac{-C_{r d}(i)}{N}+\frac{\mu}{N} P_{r}(i)\left|g_{s 2}(i)\right|^{2}+\frac{\lambda}{N} C_{r d}(i) \\
& +\frac{\gamma}{N} P_{r}(i)+\alpha_{2}(i)-\beta_{2}(i)+\varphi(i)=0,
\end{aligned}
$$

$$
\begin{aligned}
& \frac{\partial \mathcal{L}}{\partial q_{3}(i)}=\frac{-C_{r d}(i)}{N}+\frac{\mu}{N}\left(P_{s}(i)\left|g_{s 1}(i)\right|^{2}+P_{r}(i)\left|g_{s 2}(i)\right|^{2}\right) \\
& -\frac{\lambda}{N} C_{s r}^{f}(i)+\frac{\lambda}{N} C_{r d}(i) \frac{\gamma}{N}\left(P_{s}(i)+P_{r}(i)\right)+\alpha_{3}(i)-\beta_{3}(i) \\
& +\varphi(i)=0 .
\end{aligned}
$$

If $\boldsymbol{q}(i)=[1,0,0]$ then $\alpha_{2}(i), \alpha_{3}(i)$ and $\beta_{1}(i)$ will be zero based on the complementary slackness condition, and therefore, we have

$$
N\left(\alpha_{1}(i)+\varphi(i)\right)=\lambda C_{s r}^{h}(i)-\mu P_{s}(i)\left|g_{s 1}(i)\right|^{2}-\gamma P_{s}(i) \triangleq \Gamma_{1}(i),
$$

$N\left(\varphi(i)-\beta_{2}(i)\right)=(1-\lambda) C_{r d}(i)-\mu P_{r}(i)\left|g_{s 2}(i)\right|^{2}-\gamma P_{r}(i) \triangleq \Gamma_{2}(i)$, 
TABLE I

NECESSARY CONDITIONS FOR OPTIMAL $\boldsymbol{q}(i)$

\begin{tabular}{|c|c|}
\hline $\boldsymbol{q}(i)$ & Necessary conditions \\
\hline$[1,0,0]$ & $\Gamma_{1}(i) \geq 0, \Gamma_{1}(i) \geq \Gamma_{2}(i), \Gamma_{1}(i) \geq \Gamma_{3}(i)$. \\
\hline$[0,1,0]$ & $\Gamma_{2}(i) \geq 0, \Gamma_{2}(i) \geq \Gamma_{1}(i), \Gamma_{2}(i) \geq \Gamma_{3}(i)$. \\
\hline$[0,0,1]$ & $\Gamma_{3}(i) \geq 0, \Gamma_{3}(i) \geq \Gamma_{1}(i), \Gamma_{3}(i) \geq \Gamma_{2}(i)$. \\
\hline$[0,0,0]$ & $\Gamma_{1}(i) \leq 0, \Gamma_{2}(i) \leq 0, \Gamma_{3}(i) \leq 0$. \\
\hline
\end{tabular}

$N\left(\varphi(i)-\beta_{3}(i)\right)=C_{r d}(i)-\mu\left(P_{s}(i)\left|g_{s 1}(i)\right|^{2}+P_{r}(i)\left|g_{s 2}(i)\right|^{2}\right)$ $+\lambda\left(C_{s r}^{f}(i)-C_{r d}(i)\right)-\gamma\left(P_{s}(i)+P_{r}(i)\right) \triangleq \Gamma_{3}(i)$.

Based on the dual feasibility condition, $\beta_{2}(i), \beta_{3}(i), \varphi(i)$, and $\alpha_{1}(i)$ are non-negative, and thus, $\Gamma_{1}(i) \geq 0$ holds. By subtracting (30) from (29) and (31) from (29), we obtain

$$
\begin{aligned}
& \Gamma_{1}(i)-\Gamma_{2}(i)=N\left(\alpha_{1}(i)+\beta_{2}(i)\right) \geq 0, \\
& \Gamma_{1}(i)-\Gamma_{3}(i)=N\left(\alpha_{1}(i)+\beta_{3}(i)\right) \geq 0 .
\end{aligned}
$$

Therefore, the necessary conditions for $\boldsymbol{q}(i)=[1,0,0]$ are $\Gamma_{1}(i) \geq 0, \Gamma_{1}(i) \geq \Gamma_{2}(i)$ and $\Gamma_{1}(i) \geq \Gamma_{3}(i)$. Similarly, other cases of $\boldsymbol{q}(i)$ have the necessary conditions as in Table I. As can be seen from this table, the different cases of $\boldsymbol{q}(i)$ have mutually exclusive necessary conditions except when $\Gamma_{1}(i)=\Gamma_{2}(i), \Gamma_{2}(i)=\Gamma_{3}(i), \Gamma_{1}(i)=\Gamma_{3}(i)$, and $\Gamma_{1}(i)=\Gamma_{2}(i)=\Gamma_{3}(i)$. Nevertheless, because the occurrence probabilities of these situations are approximately zero, selecting either $\boldsymbol{q}(i)=[0,0,0],[1,0,0],[0,1,0]$, or $[0,0,1]$ does not alter the maximum average secondary throughput. Thus, the conditions in Table I are both necessary and sufficient which results in Theorem 1. Similarly, the maximization problem for the case of S-CSI-I can be solved by substitution of $\Psi_{1}$ and $\Psi_{2}$ by $\left|g_{s 1}(i)\right|^{2}$ and $\left|g_{s 2}(i)\right|^{2}$, respectively, as in (7).

To compute the optimum transmit power of the SS and the $\mathrm{SR}$, we set to zero the derivative of the Lagrangian function with respect to $P_{s}(i)$, and $P_{r}(i)$.

$$
\begin{aligned}
& \quad \frac{\partial \mathcal{L}}{\partial P_{s}(i)}=\left(q_{1}(i)+q_{3}(i)\right)\left(\frac{\mu}{N}\left|g_{s 1}(i)\right|^{2}+\frac{\gamma}{N}\right)-\nu_{1}(i)+ \\
& -\frac{\lambda\left|h_{s r}(i)\right|^{2}}{N \ln 2} \times\left(\frac{q_{1}(i)}{1+P_{s}(i)\left|h_{s r}(i)\right|^{2}+P_{p}\left|g_{p 1}(i)\right|^{2}}+\right. \\
& \left.\quad+\frac{q_{3}(i)}{1+P_{s}(i)\left|h_{s r}(i)\right|^{2}+P_{p}\left|g_{p 1}(i)\right|^{2}+P_{r}(i)\left|g_{r r}(i)\right|^{2}}\right)=0, \\
& \text { and } \\
& \frac{\partial \mathcal{L}}{\partial P_{r}(i)}=\left(q_{2}(i)+q_{3}(i)\right)\left(\frac{\mu}{N}\left|g_{s 2}(i)\right|^{2}+\frac{\gamma}{N}\right)-\nu_{2}(i)+ \\
& +\frac{q_{3}(i)\left|g_{r r}(i)\right|^{2} P_{s}(i)\left|h_{s r}(i)\right|^{2} \lambda}{N \ln 2\left(1+P_{r}(i)\left|g_{r r}(i)\right|^{2}+P_{p}\left|g_{p 1}(i)\right|^{2}+P_{s}(i)\left|h_{s r}(i)\right|^{2}\right)} \\
& +\frac{\left(q_{2}(i)+q_{3}(i)\right)\left|h_{r d}(i)\right|^{2}(\lambda-1)}{N \ln 2\left(1+P_{p}\left|g_{p 2}(i)\right|^{2}+P_{r}(i)\left|h_{r d}(i)\right|^{2}\right)}=0 .
\end{aligned}
$$

Suppose that at time slot $i$, the HD mode with the transmitting $\mathrm{SS}$ is selected, and hence, $q_{1}(i)=1, q_{2}(i)=q_{3}(i)=0$, and $P_{s}(i) \neq 0$ hold. Based on the complementary slackness condition, $\nu_{1}(i)=0$, and thus, by substitution of $q_{1}(i)$ and $\nu_{1}(i)$ into (34), the transmit power of the SS in the HD mode is obtained as

$$
P_{s}^{h}(i)=\left[\frac{\lambda}{\ln 2\left(\mu\left|g_{s 1}(i)\right|^{2}+\gamma\right)}-\frac{1+P_{p}\left|g_{p 1}(i)\right|^{2}}{\left|h_{s r}(i)\right|^{2}}\right]^{+} .
$$

In addition, if at time slot $i$, the SR in the HD mode is selected for data transmission to the SD, $q_{2}(i)=1, q_{1}(i)=q_{3}(i)=0$, and $P_{r}(i) \neq 0$ hold, and thus, according to the complementary slackness constraint, $\nu_{2}(i)=0$. Therefore, by substitution of $q_{2}(i)$ and $\nu_{2}(i)$ into (35), the transmit power of the SR in the HD mode can be written as

$$
P_{r}^{h}(i)=\left[\frac{1-\lambda}{\ln 2\left(\mu\left|g_{s 2}(i)\right|^{2}+\gamma\right)}-\frac{1+P_{p}\left|g_{p 2}(i)\right|^{2}}{\left|h_{r d}(i)\right|^{2}}\right]^{+} .
$$

Similarly, if FD mode is selected in the SN, $q_{3}(i)=1, q_{1}(i)=$ $q_{2}(i)=0, P_{s}(i) \neq 0$, and $P_{r}(i) \neq 0$ hold. Based on the complementary slackness condition, $\nu_{1}(i)$ and $\nu_{2}(i)$ are zero. Thus, by substituting $q_{3}(i), \nu_{1}(i)$, and $\nu_{2}(i)$ into (34) and (35), the transmit power of the SS and the SR in the FD mode is obtained as

$$
\begin{aligned}
P_{s}^{f}(i)=\left[\frac{\lambda\left|h_{s r}(i)\right|^{2}+\left|g_{r r}(i)\right|^{2}}{\ln 2\left(\mu\left|g_{s 1}(i)\right|^{2}+\gamma\right)\left|h_{s r}(i)\right|^{2}}\right. \\
\left.-\frac{1+P_{p}\left|g_{p 1}(i)\right|^{2}}{\left|h_{s r}(i)\right|^{2}+\left|g_{r r}(i)\right|^{2}}\right]^{+}
\end{aligned}
$$

and

$$
\begin{aligned}
P_{r}^{f}(i)=\left[\frac{1-\lambda}{\ln 2\left(\mu\left|g_{s 2}(i)\right|^{2}+\gamma+\left|g_{r r}(i)\right|^{2}\right)}\right. \\
\left.-\frac{1+P_{p}\left|g_{p 2}(i)\right|^{2}}{\left|h_{r d}(i)\right|^{2}+\left|g_{r r}(i)\right|^{2}}\right]^{+} .
\end{aligned}
$$

This completes the proof.

\section{REFERENCES}

[1] J. I. Choi, M. Jain, K. Srinivasan, P. Levis, and S. Katti, "Achieving Single Channel, Full Duplex Wireless Communication," in Proc. of ACM MobiCom10, pp. 1-12, 2010.

[2] G. Liu, F. Yu, H. Ji, V. Leung, and X. Li, 'In-Band Full-Duplex Relaying: A Survey, Research Issues and Challenges," IEEE Commun. Surveys Tutorials, vol. 17, no. 2, pp. 500-524, Secondquarter 2015.

[3] E. Ahmed and A. Eltawil, "All-Digital Self-Interference Cancellation Technique for Full-Duplex Systems," IEEE Trans. Wireless Commun., vol. 14, no. 7, pp. 3519-3532, Jul. 2015.

[4] M. Darabi, B. Maham, and Y. Zhang, "Buffer-Aided Link Selection for Incremental Relaying Systems," in Proc. IEEE ISCC, pp. 1-6, July 2014.

[5] N. Nomikos, T. Charalambous, and I. Krikidis, "A Buffer-Aided Successive Opportunistic Relay Selection Scheme With Power Adaptation and Inter-Relay Interference Cancellation for Cooperative Diversity Systems," IEEE Trans. Commun., vol. 63, no. 5, pp. 1623-1634, May 2015.

[6] N. Zlatanov; D. Hranilovic; J. S. Evans, "Buffer-Aided Relaying Improves Throughput of Full-Duplex Relay Networks with Fixed-Rate Transmissions," IEEE Commun. Letters, 2016, doi: 10.1109/LCOMM.2016.2606102.

[7] G. Chen, Z. Tian, Y. Gong, and J. Chambers, 'Decode-and-Forward Buffer-Aided Relay Selection in Cognitive Relay Networks," IEEE Trans. Veh. Tech., vol. 63, no. 9, pp. 4723-4728, Nov. 2014.

[8] M. Darabi, B. Maham, X. Zhou, and W. Saad, "Buffer-Aided Relay Selection with Interference Cancellation and Secondary Power Minimization for Cognitive Radio Networks," in Proc. IEEE DySPAN, Apr. 2014

[9] M. Darabi, V. Jamali, B. Maham and R. Schober, "Adaptive Link Selection for Cognitive Buffer-Aided Relay Networks," IEEE Communications Letters, vol. 19, no. 4, pp. 693-696, Apr. 2015.

[10] K. T. Phan and T. Le-Ngoc, "Power Allocation for Buffer-Aided Full-Duplex Relaying With Imperfect Self-Interference Cancellation and Statistical Delay Constraint," IEEE Access, vol. 4, no. , pp. 3961-3974, 2016.

[11] Ch. Chang and T. Zajic, "Effective Bandwidths of Departure Processes from Queues with Time Varying Capacities," in Proc. IEEE INFOCOM, 1995, pp. 1001-1009 vol. 3. 